\title{
LA DIETA DE LAS CLASES TRABAJADORAS A FINALES DEL SIGLO XIX EN GRAN BRETAÑA ${ }^{1}$ \\ WORKING-CLASS DIETS IN LATE NINETEENTH-CENTURY BRITAIN
}

\author{
Derek John Oddy* \\ University of Westminster
}

Resumen: El artículo incluye los datos de 6 estudios realizados entre 1887 y 1901, correspondientes a 151 presupuestos familiares divididos en 5 grupos de ingresos comprendidos entre familias pobres con ingresos irregulares y familias con criados. Se calculó el consumo semanal de pan, patatas, azúcar, cereales, carne y leche, además de la ingesta diaria de energía, proteínas, carbohidratos, calcio y hierro.

Se pudo constatar que los primeros 2 grupos tenían una dieta muy pobre, el tercer grupo tenía una dieta más cercana a la media, pero todavía insuficiente, y sólo se pudo observar una mejora entre los grupos de la franja superior de ingresos con respecto a todos los alimentos, especialmente la leche.

Las familias gastaban el $58 \%$ de sus ingresos en alimentos. El pan, la harina y las patatas eran los alimentos básicos entre las familias estudiadas, proporcionando el $61 \%$ de la ingesta total de energía. La carne representaba el $11 \%$ de la energía y el $29 \%$ del gasto, pudiendo observarse que el estatus de los alimentos

${ }^{1}$ Este trabajo, financiado en un principio por subvenciones a investigación procedentes de la industria, ha sido apoyado por el Consejo de Investigación de las Ciencias Sociales (Social Science Research Council). Mi agradecimiento, por su estímulo y asesoramiento, a los profesores T. C. Barker y John Yudkin, que leyeron los borradores iniciales de este artículo.

* Original publicado en inglés, The Economic History Review, 23 (2), 1970. Traducción de José M. ${ }^{\text {a }}$ Díaz. 
afectaba a su consumo, con la posibilidad de que se diesen ciertos prejuicios negativos hacia la fruta, las verduras y la leche.

Palabras clave: dieta, alimentos, ingresos, gasto, verduras, carne, leche, proteínas, carbohidratos.

\begin{abstract}
Data from 6 surveys carried out between 1887 and 1901 were analysed for 151 families budgets divided into 5 income groups, ranging from those very poor families with irregular incomes to those keeping servants. The average weekly consumption of bread, potatoes, sugar, cereals, meat and milk was calculated, as well as the daily intake of energy, protein, carbohydrates, calcium and iron.

It was found that the first 2 groups got very poor diets, the $3^{\text {rd }}$ group got a diet corresponding to average but still poor. An improvement could be observed with the 2 groups in the upper income range in all foods, particularly in milk.

Families spent $58 \%$ of their income on food. Bread, flour and potatoes were the staples among the families surveyed, providing $61 \%$ of total energy intake. Meat provided $11 \%$ of energy and $29 \%$ of expenditure. Status of foods also had an impact on intake; there may have been persistent prejudice against fruit, vegetables and milk.
\end{abstract}

Key words: diet, food, income, expenditure, vegetables, meat, milk, protein, carbohydrates. 
El análisis de los presupuestos familiares proporciona un interesante sesgo dentro del estudio de la vida de las familias trabajadoras a finales del siglo XIX. Aunque la mayor parte del material existente es sobradamente conocido, las referencias al mismo se han limitado generalmente a estimaciones sobre el alcance de la pobreza. Sin embargo, los informes que incluyen presupuestos familiares individuales son especialmente importantes como prueba fehaciente de la cantidad de alimentos que las familias trabajadoras consumían en la realidad. Entre 1887 y 1901, hubo seis informes que se incluyen en este trabajo. Ninguno de estos estudios fue realizado a gran escala ya que, a excepción de uno, todos los demás fueron el resultado de esfuerzos aislados de investigadores individuales que, en raras ocasiones, podían analizar más de veinticinco familias de cada vez. Sin embargo, considerando todos estos estudios de forma global, aparecen detallados 179 presupuestos familiares, correspondientes a los últimos años del siglo, de los que 151 resultaron ser susceptibles de análisis en términos dietéticos. ${ }^{2}$ Este número de presupuestos es, evidentemente, demasiado reducido para realizar cualquier análisis estadístico, y podría resultar bastante arriesgado intentar establecer generalizaciones sobre la vida de las clases trabajadoras en su conjunto basándose en esta evidencia. Sin embargo, la información extraída de estos presupuestos proporciona algún indicio de la dieta de diversos sectores de esta población. ${ }^{3}$ Aproximadamente el veinticuatro por ciento de los presupuestos analizados procede de los Midlands y del norte industrial de Inglaterra, incluyendo ejemplos de Manchester (5), de Sheffield (4), de las poblaciones textiles del West Riding (4), de Tyneside (9) y de los distritos mineros de Northumberland (5). De Londres proceden 28 presupuestos, el 19 por ciento del total. Otro 16 por ciento procede de ciudades provinciales no industriales de Inglaterra, entre las que destaca por su influencia predominante el informe que sobre Cork realizó Rowntree. Las ciudades escocesas aportaron también un 16 por ciento del total, y los restantes presupues-

${ }^{2}$ Los restantes 27 presupuestos no eran lo suficientemente detallados para poder realizar con ellos ningún análisis cuantitativo.

${ }^{3}$ No se ha incluido todo el material disponible. El United States Commissioner of Labor's Reports para 1890 y 1891 incluye sumarios de 1.024 presupuestos recogidos en Gran Bretaña. Sin embargo, éstos presentan dificultades, especialmente en su interpretación, y no son lo suficientemente detallados para justificar su análisis en términos nutricionales. Existen también varios presupuestos dispersos entre los informes de las sesiones de la Royal Commission on Labour sobre Agricultural Labor, 1893-4.- Parl. Papers, 1893-4 (C. 6894), xxxv. 
tos se distribuyen de forma dispersa en el grupo de trabajadores agrícolas de las zonas rurales.

Teniendo en cuenta todas estas puntualizaciones, es preciso recordar que los informes sobre la mejora de los niveles de vida a finales del siglo XIX han ido con frecuencia acompañados por referencias a un incremento en la cantidad de alimentos consumidos. El señor H. L. Beales, en su análisis de la Gran Depresión, señalaba que «se consumía el doble de té y que incluso las clases trabajadoras consumían carne importada, naranjas y productos lácteos en cantidades sin precedentes». ${ }^{4}$ El profesor Rostow también asumía que se había producido un aumento en el consumo de alimentos, con el fin de fundamentar su afirmación de que los salarios reales habían experimentado un incremento medio de un 1,5 anual a lo largo del último cuarto del siglo XIX. ${ }^{5}$ Sorprendentemente, esta interpretación contrastaba con la declaración de Sir Jack Drummond, quien afirmaba que «no sería exagerado decir que en los albores del siglo xx reinaba la malnutrición en Inglaterra como no lo había hecho antes, ni siquiera durante los períodos de gran escasez como la Edad Media o la época Tudor». ${ }^{6}$ Más recientemente, el profesor Sayers constató que, al menos con respecto a las hortalizas frescas, «existe cierta evidencia de que el aumento del número de centros urbanos durante el siglo XIX había tenido consecuencias adversas en la dieta de los ingleses». ${ }^{7}$

Por todas estas razones, cualquier tipo de evidencia que contribuya a aumentar el conocimiento de los niveles de consumo alimentario de las familias pertenecientes a las clases trabajadoras merece un análisis meticuloso.

\section{II}

Los informes aquí analizados han tenido su origen, por una parte, en la confusión existente entre diversos puntos de vista sobre el estado de la economía en la década de 1880 y, por otra parte, en el interés surgido en el resto del continente europeo por la utilización del presupuesto familiar como método de investigación. En Gran Bretaña, la difusión del folleto de

\footnotetext{
${ }^{4}$ H. L. Beales, «The «Great Depression» in Industry and Trade», Economic History Review, v (1934), 65-75.

5 W. W. Rostow, British Economy of the Nineteenth Century (1948), pp. 90-1.

6 J. C. Drummond and Anne Wilbraham, The Englishman's Food (rev. edn, 1957), p. 403. Para una discusión de este punto de vista, véase W. Ashworth, An Economic History of England, 1870-1939 (1960), pp. 251-3,

7 R. S. Sayers, A History of Economic Change in England, 1880-1939 (1967), p. 10.
} 
Andrew Mearns, ${ }^{8}$ escrito por W. T. Stead en la Pall Mall Gazzette, y los artículos de G. R. Sim's en Daily News, suscitaron inquietud entre las clases medias como lo demuestra la reacción de Charles Booth. ${ }^{9}$ A escala oficial, las opiniones de Mearns y de Sims parecen haber sido en gran medida justificadas por la Royal Commission on the Housing of the Working Classes, que constató la existencia de un grave hacinamiento y altos alquileres, especialmente en las grandes ciudades donde la falta de transporte urbano barato fue un factor agravante. Sin embargo, sólo un año más tarde, durante la depresión de 1886, el informe de la Royal Commission on Depression of Trade and Industry expresó su satisfacción ante «la inmensa mejora que ha tenido lugar en la situación de las clases trabajadoras a lo largo de los últimos veinte años» ${ }^{10}$ En estas circunstancias, la recopilación de presupuestos familiares pasó a formar parte del análisis del «problema social»o «cuestión social» que exigió los esfuerzos de los escritores política y socialmente comprometidos durante las dos últimas décadas del siglo XIX.

Estas investigaciones también supusieron un alejamiento de la forma tradicional de abordar el estudio de la prosperidad del individuo, que se había basado en el estudio de precios y salarios.

Giffen afirmó, quizás con demasiado optimismo, que existían suficientes informes sobre precios, de manera especial sobre artículos esenciales de consumo, que abarcaban largos periodos y que ilustraban el poder adquisitivo del dinero, y su punto de vista no se cuestionó. ${ }^{11}$ Sin embargo, hacia finales de la década de 1880, la influencia de investigadores sociales y de estadísticos en la Europa continental ejercía una presión considerable entre los círculos correspondientes de gran Bretaña. El profesor Leone Levi, por ejemplo, después de que Charles Booth hubiese leído su artículo sobre los pobres de Londres ante la Statistical Society, ${ }^{12}$ le solicitó que le proporcionase información basada en presupuestos para completar sus conclusiones preliminares. ${ }^{13}$ El propio Giffen consideró necesa-

${ }^{8}$ Bitter Cry of Outcast London (1883); también Contemporary Review, xliv (Dec. 1883), 924-33.

9 Véase T. S. Simey and M. B. Simey, Charles Booth Social Scientist (Oxford, 1960), pp. 64-70.

${ }^{10}$ Final Report, P. P. 1886 (C. 4893 ), xxii para 81.

11 R. Giffen, Memorandum on the Returns of Expenditure by Working Men, P. P. 1889 (C. 5861), lxxxiv.

12 C. Booth, «The Inhabitants of Tower Hamlets (School Board Division) their Condition and Occupations», Journal of the Royal Statistical Society, L (1887), 326-401.

13 Ibid. p. 394. 
rio tener en cuenta esta técnica y, en su Memorando, ${ }^{14}$ incluyó un apéndice explicativo resumiendo el trabajo llevado a cabo por Le Play y Ducpétiaux en Bélgica y Engel en Prusia. Después de la muerte de Le Play en 1882, su trabajo fue continuado por la revista Réforme Sociale y en 1883 el Director de Estadística prusiano, Ernst Engel, intentó calcular el coste del mantenimiento de un hombre, incluida la amortización de niños fallecidos antes de comenzar a generar ingresos. ${ }^{15} \mathrm{La}$ influencia de estos estudios realizados en Europa también se hizo sentir al otro lado del Atlántico. El trabajo de la Bureau of Labor Statistics en Massachussets fue citado por Giffen ante la Royal Commission on Depresión of Trade and Industry. ${ }^{16}$ En América, el trabajo de Massachussets tuvo como consecuencia el establecimiento de la United States Bureau of Labor Statistics e, indirectamente, en Gran Bretaña, la creación del Labour Department of the Board of Trade.

Sin embargo, los trabajos anteriores de Eden, ${ }^{17}$ Neild, ${ }^{18}$ y Smith,,${ }^{19}$ en los que la familia había sido considerada como la unidad económica central, se habían olvidado. La tendencia a una bajada de precios y a un incremento de los salarios pecuniarios produjo un optimismo incuestionable. El presupuesto familiar se convirtió entonces en un instrumento necesario para contrarrestar generalizaciones de este tipo y el concepto del coste de vida pasó a formar parte del vocabulario económico. Henry Higgs, cuando discutía la cuestión de los presupuestos de los trabajadores ante la Statistical Society en 1893, señalaba el vacío de conocimiento existente en esta institución, exhortando al público a que buscase entre los números de su revista durante los últimos veinte años. "Aquí, como en cualquier otra parte, afirmaba Higgs, «se refleja el quehacer estadístico de la época. Pero buscarán ustedes en vano, tratando de encontrar cualquier registro del coste total de vida de una familia o familias de cualquier tipo

${ }^{14}$ Giffen, op. cit., pp. 48-9.

15 E. Engel, Der Kostenwerth des Menschen (Berlin, 1883).

16 First Report, P. P. 1886 (C. 4621), xxi, QQ. 13, 155-161.

17 Sir Frederic Eden, The State of the Poor (1797).

18 W. Neild, «Comparative Statement of the Income and Expenditure of Certain Families of the Working Class in Manchester and Dukinfield in the Years 1836 and 1841», Jnl. R. Stat. Soc. Iv (1841-2). Para un análisis reciente de la investigación de Neild, véase J. C. McKenzie, «The Composition and Nutricional Value of Diets in Manchester and Dukinfield in 1841», Transactions of the Lancashire and Cheshire Antiquarian Society, lxxii (1962).

19 Véase Sixth Report of the Medical Officer of the Committee of Council on Health, P. P. 1864 (C. 3416), xxviii, app. 6, «Report by Dr Edward Smith on the Food of the Poorer Labouring Classes in England». 
de vida. Sin embargo, estos hechos constituyen el elemento fundamental de la condición de las personas». ${ }^{20}$

En este sentido, el Labour Department of the Board of Trade consiguió llevar a cabo el primer estudio, publicando en 1889, Returns of Expenditure by Working Men. Fue, sin duda, mucho menos de lo que se esperaba. Al intentar realizar un estudio exhaustivo mediante un cuestionario escrito, el Labour Department siguió el mismo procedimiento por medio del cual la Royal Commission on Depression of Trade and Industry había obtenido unas 280 respuestas de los sindicatos y asociaciones profesionales. ${ }^{21}$

Aunque Burnett, el Labour Correspondent, envió 730 formularios a personas individuales, a representantes de los sindicatos y a secretarios de sociedades cooperativas para su distribución entre sus afiliados, sólo se recibieron 36 respuestas. De éstas sólo 34 fueron cumplimentadas lo suficientemente bien para que fuese posible su publicación. ${ }^{22}$

Charles Booth, que reunió un equipo de investigadores, entre los que se encontraban Beatrice Webb y Hubert Llewelyn Smith, pudo conseguir 30 presupuestos de familias londinenses que fueron publicados en Life and Labour of the People. ${ }^{23}$ Aunque esta faceta de su trabajo no se repitió en estudios posteriores realizados en Londres, una serie de 36 presupuestos más, obtenidos entre 1891 y 1894, fue publicada por el «Economic Club». ${ }^{24}$ De manera similar, al final de la década, el estudio que B. Seebohm Rowntree realizó de York incluía 26 presupuestos obtenidos entre 1899 y 1901. ${ }^{25}$ Un trabajo realizado simultáneamente con el de Rowntree fue un minucioso estudio realizado en Edimburgo, que proporcionó 16 muestras de los ingresos y gastos de familias trabajadoras. ${ }^{26}$

${ }^{20}$ Henry Higgs, «Workmen's Budgets», Jnl. R. Stat. Soc. lvi (1893), 255.

${ }^{21}$ Final Report, op. cit., P. x.

22 Returns of Expenditure, op. cit., P. 4.

23 C. Booth, Life and Labour of the People, i (1889), 134-9. Booth no restringió sus estudios limitándose a las familias del East End.

${ }^{24}$ Family Budgets: being the income and expenses of twenty-eight British Households, 1891-1894. En el catálogo de B. M., el trabajo está fechado en 1896, atribuyéndose su autoría a «Miss E. Mollet \& Miss Robertson». Booth, Ernest Aves y Henry Higgs aparecieron como firmantes del informe. Algunas familias fueron estudiadas en intervalos diferentes o según cambiaban las circunstancias, arrojando un saldo total de 36 presupuestos.

25 B. Seebohm Rowntree, Poverty: A Study of Town Life (1901), pp. 394-419.

26 D. N. Paton, J. C. Dunlop, y Elsie Inglis, On the Dietaries of the Labouring Classes of the City of Edinburgh (1901). 
Otro estudio más, de singular interés, por concentrarse en el consumo individual de alimentos en lugar de referirse a la unidad familiar, fue el realizado por el doctor Thomas Oliver, Profesor de fisiología en la facultad de medicina de Newcastle-upon-Tyne, quien experimentó un procedimiento de encuesta similar al llevado a cabo por el Labour Department of the Board of Trade, enviando circulares a sus amigos médicos en diferentes partes del país. En un artículo publicado en la Fotnightly Review, ${ }^{27}$ se constató una mejor respuesta de los trabajadores que la obtenida por el Labour Department; no obstante, publicó sólo 31 muestras en el Lancet $^{28}$ en 1895. Aunque los resultados obtenidos por el doctor Oliver fueron más tarde desestimados por Paton, Dunlop e Inglis, ${ }^{29}$ éstos parecen calcular con bastante exactitud los alimentos consumidos y proporcionar un cálculo, aunque sea aproximado, de la distribución de los alimentos dentro de la unidad familiar.

\section{III}

El análisis de la información fue llevado a cabo mediante un proceso asistido por ordenador que garantizó un tratamiento estandarizado de cada presupuesto alimentario. ${ }^{30}$ Debido a la falta de información en estos estudios sobre la distribución de alimentos dentro de la unidad familiar o sobre necesidades especiales (tales como periodos de embarazo o lactancia), los resultados se presentan dividiendo los alimentos entre el número de miembros de la familia, es decir, mediante un cálculo per capita. Por tanto, las variaciones relativas a las necesidades calóricas de niños y adultos no pudieron ser tenidas en cuenta a la hora de interpretar los resultados. No obstante, aparte del valor energético de sus dietas, tanto adultos como niños tienen necesidades similares en lo que respecta a determinados nutrientes.

El consumo de alimentos se especifica en la Tabla 1. Con relación a éstos, las cifras medias de 6,7 libras de pan y 1,6 libras de patatas son los ítems mayores de la dieta. Sin embargo, estas cantidades parecen sorprendentemente pequeñas. Otro grupo amplio de dietas del siglo XIX fue

\footnotetext{
27 Fortnightly Review, Oct. 1894.

28 «The Diet of Toil», Lancet, 29 June 1895, pp. $1^{\circ} 629-35$.

29 Paton, Dunlop e Inglis, op. cit., p. 81.

30 En el apéndice se añade una nota explicativa de los problemas de análisis.
} 


\section{Tabla 1}

Consumo medio semanal de alimentos

\begin{tabular}{ccccccccc}
\hline $\begin{array}{c}\text { Bread } \\
\text { (libr.) }\end{array}$ & $\begin{array}{c}\text { Potatoes } \\
\text { (libr.) }\end{array}$ & $\begin{array}{c}\text { Sugar } \\
\text { (onz.) }\end{array}$ & $\begin{array}{c}\text { Cereals } \\
\text { (onz.) }\end{array}$ & $\begin{array}{c}\text { Fats } \\
\text { (onz.) }\end{array}$ & $\begin{array}{c}\text { Meat } \\
\text { (libr.) }\end{array}$ & $\begin{array}{c}\text { Milk } \\
\text { (pint.) }\end{array}$ \\
\hline
\end{tabular}

(a) Por estudios

1. Board of Trade, circa 1887

2. Booth, circa 1888

$\begin{array}{lllllll}7,5 & 1,6 & 15 & 3,7 & 4,9 & 1,4 & 1,5\end{array}$

3. Economic Club, circa 1891-4

$\begin{array}{lllllll}6,5 & 2,1 & 12 & 1,5 & 3,9 & 1,6 & 1,4\end{array}$

4. Oliver, circa 1894

$7,0 \quad 1,1 \quad 15$

$\begin{array}{llll}1,7 & 5,7 & 1,4 & 1,3\end{array}$

5. Rowntree, circa 1899-01

$5,5 \quad 3,6 \quad 16$

5,4

$5,2 \quad 3,2$

1,6 (sólo familias de clase trabajadora*)

$6,3 \quad 2,2$

17

2,0

6,7

1,9

2,4

$(6,8) \quad(2,0)$

(15)

$(1,7)$

$(5,2) \quad(1,5)$

$(1,5)$

6. Paton, Dunlop e Inglis, circa 1900

$4,7 \quad 1,2$

13

6,4

$4,2 \quad 1,2$

1,6 Media

$6,7 \quad 1,6$

14

2,8

$4,9 \quad 1,4$

(b) Por grupos según sus ingresos

\begin{tabular}{lrrrrrrr} 
A. Menos de 18 chelines por semana & 5,5 & 1,1 & 11 & 2,0 & 3,9 & 0,9 & 1,0 \\
B. Entre 18 y 21 chel. por semana & 6,5 & 1,6 & 16 & 1,7 & 4,4 & 1,2 & 1,2 \\
C. Entre 21 y 30 chelines por semana & 7,2 & 1,6 & 14 & 3,2 & 4,7 & 1,5 & 1,2 \\
D. Más de 30 chelines por semana & 7,3 & 2,4 & 17 & 4,2 & 6,0 & 1,8 & 2,2 \\
F. Familias con algún sirviente & 5,0 & 2,9 & 23 & 3,2 & 12,0 & 3,2 & 5,4 \\
\hline
\end{tabular}

* El estudio de Rowntree incluía seis familias de clase media que tenían algún sirviente. El nivel de vida más elevado de estas familias altera significativamente los resultados del análisis. Las familias de clase I y clase II fueron utilizadas para obtener una media separada correspondiente a la clase trabajadora, lo que se puede observar en las cifras que aparecen entre paréntesis. Únicamente estas cifras de las familias de clase trabajadora se utilizan para la media de todos los estudios.

recogido en 1862-3 por el doctor Edward Smith para el Medical Department of the Privy Council. Este informe y las investigaciones llevadas a cabo entre 1887 y 1901 no son estrictamente comparables pero los análisis del estudio de Smith dan una idea aproximada del consumo semanal per capita de pan de los trabajadores, que era de 9,1 libras entre los que lo consumían en el interior y de 11,6 libras entre los trabajadores agrícolas. ${ }^{31}$ En cuanto a las patatas, su consumo era de 2,4 y 6,4 libras respectivamente en $1863 .^{32}$ Es

31 T. C. Barker, D. J. Oddy, and Yudkin, The Dietary Surveys of Dr Edward Smith, 1862-3 (1970), p. 43.

32 Ibid. 
interesante destacar que R. N. Salaman estimó que el consumo de patatas descendió desde un máximo de 0,9 libras por día (6,3 libras por semana) en 1871, hasta 0,58 libras por día (4,06 libras por semana) en 1901,33 mientras que su cálculo del consumo per capita de pan indicaba que éste permaneció estable durante el periodo comprendido entre 1870 y 1889 , a 1,05 libras diarias (7,35 por semana) y descendió ligeramente durante la década de 1890, a 1,04 libras (7,28 libras por semana).

Una tendencia que ha sido generalmente aceptada, en lo que respecta al consumo de alimentos, es el incremento en el consumo de azúcar. ${ }^{34}$ Este análisis por familias de la década de 1890 sugiere que se consumía una cantidad de azúcar de 14 onzas semanales. En el estudio de Smith realizado en la década de 1863, los trabajadores de interior y los trabajadores agrícolas consumían 7,9 y 6,6 onzas semanales respectivamente. ${ }^{35}$

El nivel medio de carne consumida, según estos estudios de la década de 1890 era de 1,4 libras por semana. ${ }^{36}$ Sin embargo, otros productos de origen animal se consumían sólo en pequeñas cantidades. Se bebían menos de 1,5 pintas de leche semanales y sólo se consumían 5 onzas de grasas. Con respecto a estos dos últimos productos, las dietas resultantes de estos estudios proporcionaron pocos datos sobre el consumo de leche condensada o de margarina y es posible que estos alimentos fuesen mucho menos importantes de los que en un principio se había pensado. ${ }^{37} \mathrm{La}$ estimación de consumo de R. H. Rew en 1904 apuntaba hacia un consumo no superior a 1,5 libras de leche condensada y 4 libras de margarina por persona anuales. ${ }^{38}$

Los valores nutritivos de las dietas se muestran en la Tabla 2. La ingesta media de calorías para estas clases trabajadoras se situaba exactamente

${ }^{33}$ R. N. Salaman, The History and Social Influence of the Potato (Cambridge, 1949), pp. 539, 610-13.

${ }^{34}$ B. R. Mitchell y P. Deane, Abstract of British Historical Statistics (Cambridge, 1962), pp. 355-8.

35 Barker, Oddy y Yudkin, op. cit., p. 43.

${ }^{36}$ R. H. Rew estimó que el consumo medio de carne de los trabajadores del campo era de 1,65 libras semanales a finales del siglo.- Report of the Royal Commission on the Supply of Food and Raw Material in Time of War, P. P. 1905 (Cd. 2643), xxxix, QQ. 9534-6.

37 Cf. Ashford, op. cit., p. 252, y Drummond y Wilbraham, op. cit., pp. 332, 377, para los efectos de la leche condensada; también ibid. P. 406, donde sólo se analiza una dieta hipotética para la comparación de la mantequilla y la margarina.

${ }^{38}$ R. H. Rew, «Third Report from the Committee Appointed to inquire into Statistics available as a Basis for estimating the Production of Meat and Milk in the United Kingdom», Jnl. R. Stat. Soc. Lxvii (1904). 


\section{Tabla 2}

Ingesta media de nutrientes por día

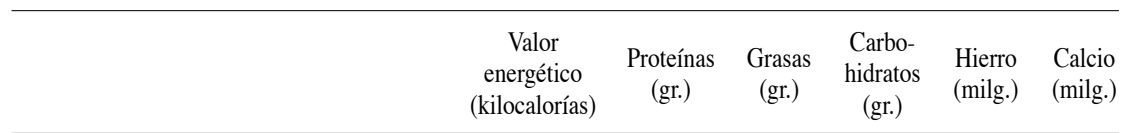

(a) Por estudios

1. Board of Trade, circa 1887

2. Booth, circa 1888

\subsection{0}

2.020

3. Economic Club, circa 1891-4

4. Oliver, circa 1894

\subsection{1}

2.799

2.307

5. Rowntree, circa 1899-01 (Sólo fam. de clase trabajadora*)

6. Paton, Dunlop e Inglis, circa 1900 Media

C. Entre 21 y 30 chel. por semana

D. Más de 30 chel. por semana

2.537

F. Familias con algún sirviente

$\begin{array}{rrrrr}59 & 57 & 372 & 10,3 & 0,31 \\ 61 & 57 & 315 & 10,6 & 0,28 \\ 54 & 60 & 335 & 9,1 & 0,27 \\ 93 & 114 & 366 & 15,5 & 0,50 \\ 65 & 72 & 350 & 11,4 & 0,44 \\ (57) & (59) & (328) & (10,1) & (0,31) \\ 54 & 52 & 276 & 10,1 & 0,38 \\ 57 & 57 & 337 & 10,0 & 0,30\end{array}$

* Véase nota en Tabla 1.

por debajo de las 2.100 kilocalorías. ${ }^{39}$ A pesar de que existiese la posibilidad de que el consumo de leche y de carne hubiese aumentando a finales del siglo XIX, la ingesta de calorías era todavía poco elevada, una media de 58 gramos por día, mientras que la cantidad de carbohidratos reflejaba

39 Durante la década de 1960, el valor energético de la dieta en Gran Bretaña era de unas 2.600 kilocalorías por persona y día. Sin embargo, en el caso de las familias de la clase trabajadora con más miembros (Las clases sociales C y D1 con tres niños), lo que es quizás una comparación más exacta con estas familias en la década de 1.890, la ingesta diaria era solamente de 2.200 kilocalorías. Véase Household Food Consumption and Expenditure: 1965 (1967), Table 26, p. 88, Table 31, p. 94. Durante la década de 1930, se calculaba que en el rango más bajo del 10 por ciento el valor energético de la dieta era de 2.317 kilocalorías por persona y día. Véase J. B. Orr, Food, Health and Income (1936), Table vii, p. 40. 
un descenso en el consumo de pan. Las cifras correspondientes al calcio eran también sumamente bajas.

Por otra parte, tanto en la Tabla 1 como en la Tabla 2, las familias han sido reagrupadas según sus niveles de ingresos con el fin de determinar el estado nutricional específico de cada grupo. ${ }^{40}$ La parte esencial de las argumentaciones de Booth y de Rowntree consistía en determinar la línea divisoria de la pobreza, que dependía del tratamiento dado a los datos. Cada uno de los tres estudios de Booth, Rowntree y de Paton, Dunlop e Inglis adoptaron clasificaciones diferentes según los grupos de ingresos de las familias que estudiaron, pero se utilizó el sistema de Rowntree y se colocaron las familias en grupos según esta base, utilizando sus divisiones según ingresos, a la vez que su concepto de «familia regular». ${ }^{41}$ Existe un patrón evidente de consumo notablemente similar para todos los alimentos y nutrientes. En estos niveles de ingresos, el consumo de alimentos se incrementa al aumentar los ingresos. Esta tendencia era más acusada para la leche, la carne y la mantequilla, lo cual se demuestra, como cabía esperar, mediante los análisis de proteína y grasa. En cuanto a los alimentos feculentos, tales como el pan, los cereales y las patatas, la tendencia era

40 El estudio del doctor Oliver excluyó ya que los niveles de ingresos eran prácticamente desconocidos.

${ }^{41}$ Rowntree, op. cit., p. 82. Rowntree asumía un tipo de familia de entre cuatro y seis miembros, basándose en el tamaño medio (4,73 personas) en el Censo de 1891.

Grupos de Ingresos:

El Grupo A corresponde a familias que percibían menos de 18 chelines por semana como ingresos totales familiares. La clasificación de las familias pobres con ingresos irregulares correspondía groso modo con este grupo. Booth no proporcionó ejemplos de su clase A la que el describía como marginados sociales y trabajadores ocasionales de baja categoría. Sin embargo, algunos ejemplos de su clase B, que sólo percibían ingresos de forma ocasional, fueron incluidos como inferiores a este nivel de ingresos.

El Grupo B está basado en unos ingresos de entre 18 y 21 chelines semanales y abarca los niveles más bajos de los trabajadores sin cualificaciones.

Las familias del Grupo $C$ percibían ingresos de entre 21 y 30 chelines por semana y constaban principalmente de trabajadores que percibían «ingresos estándar regulares». Booth, Labour and Life, i, p. 50.

El Grupo D está compuesto por familias de trabajadores cualificados con unos ingresos por exceso de 30 chelines por semana, aunque algunas familias con hijos mayores que realizaban trabajos remunerados podrían alcanzar unos ingresos totales similares para poder ser incluidos en este grupo.- Rowntree, op. cit., p. 43.

El Grupo E consta de familias que, en la investigación de Rowntree, tenían algún sirviente. Este grupo tiene un valor estadístico limitado, al representar un número muy pequeño. 
menos acusada y, con respecto a dos de estas categorías alimentarias (el pan y los cereales), las familias de clase media, que tenían algún sirviente, de hecho consumían menos cantidad que las familias más acomodadas dentro de las clases trabajadoras.

$\mathrm{Si}$ se analizan los grupos de ingresos por separado, ambos grupos A y B proporcionan ejemplos de las dietas que Rowntree situaba en la categoría de pobreza primaria. ${ }^{42}$

No existe duda alguna sobre la pobreza de la dieta: los individuos pertenecientes a estos grupos ingerían sólo 6 libras de pan a la semana y la dieta total proporcionaba menos de 2.000 kilocalorías al día. Por regla general, su dieta era similar a la del grupo más desfavorecido (las costureras) entre los analizados por Edward Smith en $1863,{ }^{43}$ con la excepción del consumo de azúcar, que era el doble que el de la década de 1860.

Con respecto al grupo $\mathrm{C}$, el consumo de alimentos y nutrientes correspondía con gran exactitud con la media correspondiente a la clase trabajadora, calculada a partir de la media de cada investigación. El diferencial nutricional existente entre los grupos B y C era insignificante y parece evidente que, en palabras del propio Rowntree, «no pocos de aquellos individuos pertenecientes a esta clase vivían en un estado de pobreza». ${ }^{44}$ No se producía una mejora en estándares en el grupo D, hasta que los ingresos excedían los 30 chelines. Esto se hizo patente en el consumo de todos los alimentos pero, de manera más significativa en el consumo de la leche, que llegó a superar las dos pintas semanales por persona.

Sigue siendo una tarea difícil evaluar la suficiencia de estas dietas en términos nutricionales debido a la falta de evidencia sobre la distribución de alimentos dentro de la familia. Se supone, sin embargo, que el hombre generalmente recibía la parte del león de los alimentos disponibles (especialmente la carne), pero esto es difícil de justificar. La evidencia correspondiente a las dietas individuales de hombres y mujeres recogida por el doctor Oliver, aunque no es concluyente, aporta cierta base que permite comparaciones de ingesta alimentaria. Esto aparece reflejado en la Tabla 3.

42 Ambos grupos A y B percibían ingresos por debajo del nivel de los 21 chelines y 8 peniques, en el que Rowntree trazó la línea de pobreza para una familia moderada. Vide Rowntree, op. cit., ch. iv.

43 Barker, Oddy, and Yudkin, op. cit., p. 43.

44 Rowntree, op. cit., p. 61. 


\section{Tabla 3}

Ingesta diaria de nutrientes según sexo

\begin{tabular}{lcccccc}
\hline & $\begin{array}{c}\text { Valor energético } \\
\text { (kilocalorías) }\end{array}$ & $\begin{array}{c}\text { Proteínas } \\
\text { (gr.) }\end{array}$ & $\begin{array}{c}\text { Grasas } \\
\text { (gr.) }\end{array}$ & $\begin{array}{c}\text { Carbohidratos } \\
\text { (gr.) }\end{array}$ & $\begin{array}{r}\text { Hierro } \\
\text { (milg.) }\end{array}$ & $\begin{array}{c}\text { Calcio } \\
\text { (milg.) }\end{array}$ \\
\hline Hombres & 3.321 & 114 & 146 & 387 & 19,0 & 0,69 \\
Mujeres & 1.870 & 48 & 51 & 310 & 7,9 & 0,18 \\
\hline
\end{tabular}

Debido al reducido número de individuos, fue necesario ignorar sus referencias relativas a las dietas de los jóvenes y los casos de dietas de hambre. La única evidencia, por tanto, corresponde a 21 hombres y 6 mujeres, que arrojan una ingesta media ligeramente superior a las 3.330 kilocalorías diarias para los hombres y 1.870 para las mujeres. Las causas de estas diferencias son evidentes. El consumo de grasas entre los hombres era el triple que el de las mujeres, a la vez que el consumo de proteínas entre los hombres era también el doble que entre las mujeres. Esencialmente, la dieta de las mujeres consistía en pan y té, mientras que los hombres consumían una comida principal consistente en carne, bacon o pescado y patatas. Sin embargo, la información proporcionada por el doctor Oliver deja sin resolver la cuestión principal: ¿Hasta qué punto eran adecuadas estas dietas para la población de la década de 1890? Desde la perspectiva contemporánea, expresada por Charles Booth, los grupos de rentas más bajas o «"muy pobres" están en todo momento, más o menos, "en estado de necesidad", malnutridos y mal vestidos». ${ }^{45}$ Los ingresos percibidos por los trabajadores sin cualificaciones eran «insuficientes para proporcionar alimentos, vivienda y ropa adecuados para mantener a una familia de tamaño moderado en unas condiciones de mínima subsistencia física». ${ }^{46}$ Y Rowntree enfatizaba: «Todo trabajador que tenga tres hijos se ve obligado a pasar un tiempo, probablemente de unos diez años, durante el cual se hallará en una situación de "pobreza primaria", es decir, tanto él como su familia estarán subalimentados». ${ }^{47}$ Es imposible explicar cómo, con una dieta de 2.000 kilocalorías y 50 gramos de proteína al día se podía hacer frente de manera adecuada a las necesidades fisiológicas del ca-

\footnotetext{
45 Booth, Labour and Life, i, p. 131.

46 Rowntree, op. cit., p. 133.

47 Ibid., p. 135.
} 
beza de familia, esposa y e hijos en desarrollo. Booth confirmó la evidencia de Oliver en cuanto a la distribución de alimentos dentro de la familia: con respecto a las familias que percibían menos de 21 chelines semanales afirmaba: «de manera especial, las mujeres y los niños consumen cantidades considerables de pan y de té». ${ }^{48}$ Por otra parte, es más que probable que la carencia de conocimientos culinarios y la falta de estándares sociales hiciesen que las comidas fuesen irregulares y desequilibradas. Varios investigadores constataron hábitos de dejadez: «siempre quedan dispersos restos de comida - es probable que no exista aparador - probablemente haya un trozo de pan sobre la mesa con algo de mantequilla y un cuchillo manchado, o una tetera, una o dos tazas usadas y un plato escachado»; ${ }^{49}$ o «los niños toman té cinco o seis veces al día y se les da un trozo de pan cada vez que lo piden». ${ }^{50}$

Sin embargo, las dietas de los grupos A y B representaban una minoría dentro de la clase trabajadora. La dieta del grupo $\mathrm{C}$, dentro de la franja comprendida entre los 21 y los 31 chelines semanales, era, en palabras de Booth, «más representativa que ninguna otra del "modo en que vivimos ahora"». ${ }^{51}$

Desde su punto de vista, los ingresos regulares proporcionaban el incentivo necesario para el movimiento social ascendente:

Las comidas son más regulares. Para comer, se exigen carne y verduras todos los días. El bacon, los huevos y el pescado también encuentran su lugar en otras ocasiones. Los puddings y las tartaletas se consumen con frecuencia y el pan deja de ser el alimento básico. La destreza en la cocina se hace muy importante y, aunque susceptible de mejora, no es algo de lo que se adolezca en general. En esta clase, nadie anda escaso de alimentos. ${ }^{52}$

Su interpretación no parece consistente en el análisis de las dietas del grupo $\mathrm{C}$, que se muestra en la Tabla 2, que apunta hacia una semejanza ya comentada entre las dietas de los grupos B y C. Las familias del grupo C, con una ingesta per capita de 2.100 kilocalorías y 58 gramos de proteína, obtenía solamente 150 kilocalorías y 7 gramos de proteína más que el

\footnotetext{
48 C. Booth, Life and Labour, 2nd ser. V (1903), p. 327.

49 Ibid. p. 326.

50 Paton, Dunlop, and Inglis, op. cit., p. 17.

51 Booth, Life and Labour, v, 329.

52 Ibid.
} 
grupo B. Por tanto, la mejora en estándares sociales no se reflejaba en el estado nutricional. La brecha entre dietas suficientes e insuficientes habría que buscarla, sin embargo, entre las familias del grupo $\mathrm{C}$ - los trabajadores sin cualificaciones con empleo regular - y las del grupo D que, en términos de ingresos, eran equivalentes a las familias de los trabajadores cualificados. Una ingesta diaria de más de 2.500 kilocalorías y 70 gramos de proteína en las familias del grupo D ponía de manifiesto una notable diferencia en status nutricional entre los grupos $\mathrm{C}$ y D.

\section{IV}

Este análisis de las dietas correspondientes a la década de 1890 puede añadir peso a la suposición de que un número considerable de personas estaban deficientemente nutridas durante los últimos años del reinado victoriano. A pesar de la descripción de Booth del «modo en que vivimos ahora», el pan y las patatas constituían la comida básica en las dietas de estas familias. Tampoco existían muchas posibilidades de mejora para familias que, por término medio, ya gastaban el 58 por ciento de sus ingresos en alimentos. El dinero gastado en alimentos como el pan, la harina y las patatas producía energía de manera económica pero tenía como consecuencia una dieta pesada y, en estas familias, más del 61 por ciento de las calorías procedían de estas fuentes de carbohidratos. Sin embargo, en términos económicos, el dinero gastado en pan y harina constituía solamente el 20 por ciento del gasto total en alimentos. Por otra parte, aunque la carne suponía el 29 por ciento del gasto total alimentario, en términos de valor energético de la dieta, producía el 11 por ciento de las calorías.

Por tanto, estas familias podrían haber obtenido energías de modo más económico gastando menos en carne y más en alimentos feculentos como manifestaban las clases medias contemporáneas en sus opiniones. El Comité Interdepartamental de Deterioro Físico manifestaba: «No cabe duda de que, con más conocimientos, los pobres podrían vivir de forma más económica de lo que lo hacen pero, al igual que las demás clases, la tendencia parece ser gastar lo menos posible en alimentos». ${ }^{53}$ Esto, según el Comité, estaba relacionado con «un deseo de algún tipo de sensación,

${ }^{53}$ Report, P. P. 1904 (Cd. 2175), xxxii, para. 224.

Historia Contemporánea 48: 71-90 
comparable al que proporciona dieta de encurtidos y vinagre». ${ }^{54} \mathrm{Sin}$ embargo, esta actitud no tiene en consideración la necesidad de que la comida sea apetitosa.$^{55}$ En una dieta rica en alimentos con carbohidratos, tales como los que consumían estas familias en la década de 1890, el sabor era la consideración que imperaba de forma abrumadora a la hora de comprar alimentos. Por otra parte, el status de los alimentos también influía en el gasto alimentario y en la elección de la dieta. Sabemos poco de la situación de status de los alimentos durante el siglo diecinueve, pero existen algunos indicios de la existencia de ciertos prejuicios hacia la fruta, las verduras y la leche que perduraron hasta el comienzo del siglo veinte. ${ }^{56}$

La investigación de estas familias trabajadoras en la década de 1890 contribuye a explicar la evidente paradoja existente entre el aumento en salarios reales y el bajo estándar de nutrición y salud que los observadores percibían a finales del siglo XIX. El estudio puede sugerir que no existe la certeza de que el aumento de salarios reales o la bajada de precios de los alimentos tuviese como consecuencia un aumento del consumo de los mismos, una argumentación que ignora factores medioambientales, fisiológicos y psicológicos en la vida de las clases trabajadoras. Quizás el indicador más sensible de la salud general y de las condiciones de vida de una población sea el índice de mortalidad infantil; y éste permaneció consistentemente elevado durante la década de 1890. Aunque de forma indirecta, este hecho proporciona una observación significativa de las dietas de este periodo.

Queen Elizabeth College, Londres.

\section{Apéndice. Método de análisis}

Se utilizó un programa informático de análisis diseñado por el Departamento de Nutrición del Queen Elizabeth College. ${ }^{57}$ Fue necesario realizar ciertos cálculos antes del análisis de la dieta. Se asumió que todos los

${ }^{54}$ Ibid. para. 225.

55 Vide J. Yudkin, «Patterns and Trends in Carbohydrate Consumption and their relation to Disease», Proceedings of the Nutrition Society, xxiii, 149.

56 Drummond, op. cit., p. 379. Véase también W. B. Cheadle, Artificial Feeding and Food Disorders of Infants (1902), p. 46; L. E. Holt, The Diseases of Infancy and Childhood (New York, 1902), pp. 213-14; F. W. Pavy, A Treatise on Food and Dietetics (1875), pp. 283-311.

57 Diseñado por Mr D. S. Miller y Miss Pamela Mumford. 
pesos de los alimentos eran los del momento de la compra y que era necesario atribuir cierto margen a las pérdidas producidas durante la preparación o cocinado. En el caso de las verduras y de la carne, se dedujo un 10 por ciento de pérdida y un 20 por ciento en el caso del pescado. De forma análoga, fue necesario asumir otros aspectos con respecto a la calidad de los alimentos, ya que el análisis se realizó basándose en tablas modernas de análisis alimentario. ${ }^{58}$ Se evitó utilizar alimentos enriquecidos actuales, tales como el pan y, en su lugar, se analizó un peso equivalente de harina. En el caso en que no hubiese detalles disponibles con respecto al tipo de carne, pescado o verdura, fue necesario asumir alimentos específicos. Algunas investigaciones consideraron como hortaliza y fruta estándar al repollo y a la manzana respectivamente y así se procedió a lo largo de todo el presente análisis. Por otro lado, el eglefino (un pescado blanco muy económico del que existían abundantes datos de análisis) fue considerado el pescado estándar y para los análisis de grasa y nitrógeno relativos a la carne, fueron considerados la parte superior de la pata de ternera asada (topside), la chuleta de cordero y el cerdo asado. ${ }^{59}$

Los alimentos fueron analizados en estado crudo, resultando ser ésta la solución más práctica debido a la falta de información sobre las prácticas culinarias. No se realizó una evaluación del contenido vitamínico de las dietas al considerarse poco probable la obtención de unos resultados realistas.

Fue necesario excluir familias que no habían permanecido estables en el número de miembros durante el estudio, además de cualquier gasto significativo en comidas que hubiesen tenido lugar fuera del hogar.

\section{Conclusión $^{60}$}

Mi artículo titulado «Working-Class Diets in Late Nineteenth Century Britain» fue escrito hace más de 40 años, mucho antes de que existiesen ordenadores personales o Internet. Es, por tanto, necesario explicar cómo debe aproximarse el lector a este texto. Fue un ejemplo muy temprano de

58 R. A. McCance y E. M. Widdowson, The Composition of Foods (1960).

59 Llevados a cabo por Mr D. S. Miller.

${ }^{60}$ Con el fin de actualizar su artículo y por deseo expreso del autor, Derek John Oddy, Catedrático Emérito de Historia Social y Económica en la University of Westminster, London, se incluye este apartado en la versión española para su publicación en Historia Contemporánea. 
análisis asistido por ordenador de una gran conjunto de datos llevado a cabo en el macroordenador de la Universidad de Londres en el que los investigadores científicos del Departamento de Nutrición del Queen Elizabeth College (en la actualidad, King's College de Londres) habían instalado un conjunto exhaustivo de tablas de análisis. No se les permitía a los investigadores el acceso individual a los macroordenadores. Era necesario estandarizar todos los datos que se iban a procesar, lo que dio lugar a muchas discusiones, y se realizaron cálculos manuales con el fin de proceder a analizar con precisión alimentos del pasado mediante tablas modernas de alimentos. En el Departamento de Nutrición nadie había intentado con anterioridad asignar valores nutricionales a materiales de fuentes históricas. Por ejemplo: ¿cuánta carne tenían los canales de oveja en el siglo diecinueve? Sin duda, mucha más que la que tienen hoy, pero ¿qué valores deberían atribuírsele a la carne con respecto a la energía y a los nutrientes? Se explican en el Apéndice algunas de estas asunciones necesarias.

Cuando todos los datos fueron consignados como códigos alimentarios estándar, fueron entregados a un técnico para que los convirtiese en una cinta mecánicamente legible y así introducirla en el ordenador central. Con suerte, dos o tres días más tarde, las impresiones de los resultados sin analizar aparecían en tu mesa. Tenían que ser clasificados de tal manera que se pudiesen calcular medias correspondientes a los diferentes estudios, grupos y zonas geográficas. El trabajo en el que se basó este artículo, publicado en la Economic History Review, constituyó la primera fase de mi Tesis Doctoral.

El Departamento de Nutrición estaba interesado en un estudio longitudinal de los cambiantes hábitos alimentarios durante el periodo de la industrialización británica. Las tendencias estaban basadas en un análisis de consumo alimentario registrado en estudios de presupuestos familiares realizados entre finales del siglo dieciocho y los comienzos de la Nacional Food Survey, en la década de 1940. Un estudio de esta investigación se puede encontrar en The Cambridge Social History of Britain 1750-1950, volume 2, People and Their Environment, ch. 5, D. J. Oddy, «Food, Drink and Nutrition». Un comentario correspondiente a la segunda mitad del siglo veinte se puede encontrar en Fifty Years of the Nacional Food Survey, pero se puede observar una perspectiva más amplia en mi obra, From Plain Fare to Fusion Food, British Diet from the 1890s to the 1990s. Ésta última comienza relacionando el siglo veinte con periodos anteriores dentro del siglo diecinueve. Ningunos de estos estudios mencionados se remonta a las primeros estudios de presupuestos familiares reali- 
zados en Gran Bretaña, recopilados y publicados por el Reverendo David Davies en 1795 y por Sir Frederic Morton Eden en 1796; ambos estaban preocupados por la pobreza durante el periodo de las guerras contra Francia. El estudio y algunas discusiones sobre el análisis de presupuestos familiares se pueden encontrar en mi capítulo, el número 6, en Malcolm Smith (ed.) Human Biology and History.

Estos estudios proporcionan evidencia cuantitativa de los alimentos que consumían las clases trabajadoras en épocas diferentes, dentro de la evolución de la sociedad británica moderna. Hay algunos estudios, aunque no muchos, de las familias más acomodadas. ¿Qué credibilidad se les debe otorgar a estos trabajos? Uno de los problemas es que, aunque esta evidencia es más valiosa que la historia descriptiva, escrita por los «historiadores de la alimentación», constituye una base limitada sobre la que puedan extraer generalizaciones. La complejidad de las técnicas analíticas ha hecho que los historiadores prefieran la historia antropométrica; apoyándose, por tanto, en la estatura final de los adultos como indicador equivalente al status nutricional. Esto introduce automáticamente factores no cuantificables relativos al medio ambiente y a la salud durante el desarrollo físico, que no pueden ser desagregados de la ingesta alimentaria ni del estatus nutricional.

\section{Bibliografía}

R. A. McCance \& E. M. Widdowson, The Composition of Foods, London, her Majesty's Stationary Office, 1960.

D. J. Oddy, «The Working-Class Diet, 1886-1914», University of London, Ph D unpublished thesis, 1971.

F. M. L. Thompson (ed.), The Cambridge Social History of Britain 1750-1950, volume 2, People and their Environment, Cambridge, 1990, ch. 5, D. J. Oddy, «Food, Drink and Nutrition».

Ministry of Agriculture, Fisheries and Food, Fifty Years of the National Food Survey, London, HMSO, 1991.

D. J. Oddy, From Plain Fare to Fusion Food, British Diet From the 1890s to the 1990s, Woolbridge, The Boydell Press, 2003.

D. Davies, The Case of Labourers in Husbandry, Bath and London, Robinson, 1795.

F. M.Eden, The State of the Poor, 3 vols., London, White, 1791.

M. Smith (ed.), Human Biology and History, London, Taylor \& Francis, 2002. 